\title{
LA FÉMINISATION LINGUISTIQUE EN MILIEU FRANCOPHONE
}

\author{
Martin Pleško
}

\begin{abstract}
This paper analyses sociolinguistic situation and the position of the feminine profession words in four French-speaking countries: Belgium, France, Canada and Switzerland. The linguists have been dealing with feminization of French language since the 1970s but there has been no detailed comparative analysis of situation in the given countries so far. Although these four countries influence each other in linguistic feminization but the privileged position of feminine nouns in Quebec does not contribute to their higher usage in the European French-speaking countries.

Keywords: Belgium; France; Quebec; Switzerland; linguistic feminization; profession.

Résumé : L'article analyse la situation sociolinguistique et la position des appellations professionnelles au féminin dans quatre pays francophones - la Belgique, la France, le Canada et la Suisse. La féminisation linguistique est un sujet d'actualité depuis les années 70, mais jusqu'à présent, il n'existe pas d'analyse comparative de la situation dans les pays cibles. Il est à supposer que ces quatre pays s'influencent mutuellement. Toutefois la position privilégiée des féminins au Québec ne contribue guère à l'intensification de leur usage dans les pays francophones en Europe.
\end{abstract}

Mots-clés : Belgique ; France ; Québec ; Suisse ; féminisation linguistique ; professions.

\section{Introduction}

Notre attention portera sur la féminisation linguistique dans quatre pays francophones - la Belgique (la Communauté française de Belgique), la France, la Suisse (la Suisse romande) et le Canada (le Québec) en nous concentrant sur les noms de métiers, fonctions, grades et titres. La féminisation linguistique est étudiée des points de vue lexicale (la féminisation lexicale) et textuelle (la féminisation des textes et discours). La féminisation lexicale consiste à la formation du féminin à partir des noms masculins à l'aide des suffixes, déterminants, adjonctions lexicales ou la reprise pronominale dans la phrase suivante. La féminisation des textes a pour but d'éliminer le langage sexiste qui est d'une façon humiliante centré contre l'un des sexes, le plus souvent contre les femmes.

Dans les pays francophones, la féminisation linguistique est l'objet d'intérêt depuis quelques décennies déjà : en France depuis la mise en place de la commission de terminologie chargée de la féminisation des noms de métier présidée par Mme Benoîte Groult en 1984 et au Canada depuis 1979, année de publication des recommandations concer-

Romanica Olomucensia 24.2 (2012): 151-158 
nant la féminisation dans la Gazette officielle du Québec, 28 juillet 1979. De nombreuses études ont été réalisées, mais jusqu'à présent, il n'existe pas une analyse comparative de la féminisation de la langue dans les pays où la langue officielle est le français.

Certains considèrent le terme féminisation linguistique politiquement incorrect parce qu'il met en évidence la différence sociale entre les femmes et les hommes. Nous l'employons pour mettre des cadres précis à notre recherche et sans aucune signification péjorative.

\section{La féminisation linguistique}

La féminisation est une revendication dont le but est de parvenir à l'égalité des femmes dans tous les domaines sociaux, notamment dans la vie professionnelle, ${ }^{1} \mathrm{~d}^{\prime}$ où son caractère pluridisciplinaire. Elle est l'objet d'études de l'histoire, du droit et de la politique, de la sociologie, de la linguistique et de la sociolinguistique, de l'économie, de la culture. $C^{\prime}$ est un point de départ pour les recherches sur le genre social. ${ }^{2}$ Elle devient aussi l'objet d'études de la didactique des langues étrangères. Pour étudier la féminisation, d'un point de vue global et/ou particulier, il est indispensable de prendre conscience de sa complexité et de la considérer comme un tout.

L'idée de l'égalité des chances est propagée depuis longtemps par les moyens publics comme l'éducation, les lois, les médias, etc. Mettons en relief le fait que la capacité humaine est depuis toujours la base essentielle de l'économie. Tous les obstacles à la vie professionnelle des gens peuvent aboutir à ne pas profiter pleinement de leur capacité sur le marché du travail. Un cas de figure sont les femmes et les appellations professionnelles. Les femmes exercent aujourd'hui les mêmes professions que les hommes, par exemple l'avocat, le médecin, le professeur, les dirigeants. C'est une réalité quotidienne. L'objectif de la féminisation linguistique est de désigner d'une façon objective la réalité sociale, de refléter des changements sociaux dans la langue (Larivière 2001 : 15). Dans le cas contraire, les femmes deviennent invisibles dans la société ce qui peut susciter des stéréotypes de genre et sociaux dont les conséquences sont souvent terribles.

Le phénomène de féminisation des substantifs offre l'un des plus importants exemples des changements qu'une langue peut subir. Il est motivé par la réalité sociale, à savoir par l'accès des femmes à des secteurs professionnels qui, pendant longtemps, n'ont été occupés que par des hommes.

La langue et la société évoluent simultanément et ne peuvent pas fonctionner l'une sans l'autre. La langue est un moyen de communication qui se modifie dans les situations de communication grâce à ses locuteurs et leurs objectifs, à l'usage quotidien et au contexte socioculturel. En d'autres termes, la langue s'adapte à la réalité sociale (Labrosse 2002 : 20). Le contexte socioculturel varie selon le pays. Par là, la féminisation linguistique est une preuve que la même langue officielle ne garantit pas une évolution identique dans des territoires différents. Des formes féminines couramment utilisées au Québec ne sont pas acceptées en France; les différences sont aussi à l'intérieur d'un pays. Par exemple, en France le masculin un directeur est fréquemment employé aussi pour parler d'une femme à un haut poste alors que le féminin une directrice n'est réservé

\footnotetext{
1 Il ne s'agit pas d'un mouvement féministe. Les hommes s'engagent aussi bien que les femmes mais ils sont moins nombreux.

2 Le genre social est également désigné par l'anglicisme gender (gender studies).
} 
qu'aux directrices des établissements scolaires. Au Québec, les deux formes sont équivalentes et régulièrement utilisées.

Aujourd'hui, les utilisateurs de la langue française ont l'accès à de nombreux ouvrages spécialisés visant à intensifier l'usage des féminins et donc à respecter la place des femmes dans la société moderne. Néanmoins, c'est aux utilisateurs de la langue française de choisir quel genre ils vont utiliser. Divers facteurs peuvent agir sur leur décision, par exemple le refus de la place des femmes dans la société et de nouveaux substantifs féminins. Il est à supposer que la féminisation linguistique peut aider à atteindre l'égalité absolue des femmes et des hommes dans la société. Du point de vue social, l'importance de la féminisation linguistique est omniprésente. Il ne s'agit pas d'un mode passager ni d'un programme politique mais de la revendication fondamentale de chaque individu dans la société parce que ce qui n'est pas nommé, n'existe pas (Mathieu - Pierrel 2009 : 114). Le besoin de féminiser peut être illustré par l'exemple suivant où le masculin un chirurgien désignant une femme peut être trompeur surtout pour les utilisateurs francophones dont le français n'est pas la langue maternelle.

Un homme et son fils roulent, un soir d'automne, sur une route de campagne jonchée de feuilles mortes. Un violent orage éclate. La voiture dérape et s'écrase contre un arbre. Le père est tué sur le coup ; le fils est emmené aux urgences de l'hôpital le plus proche. Le chirurgien entre dans le bloc opératoire, s'approche du blessé et s'exclame : C'est mon fils, je ne peux pas l'opérer.

L'aperçu historique de la féminisation linguistique prouve qu'à l'époque des féminins existaient et étaient systématiquement employés. Le latin, auquel le français a emprunté la majorité de son vocabulaire, féminisait tout à l'aide des suffixes féminins, par exemple $-u s /-a$,-tor/-trix ; medicus (un médecin) $\rightarrow$ medica (une médecin), auctor (acteur) $\rightarrow$ auctrix (actrice) (Cerquiglini 1999 : 10). La question qui se pose est pourquoi des substantifs féminins n'ont pas été maintenus ? C'est suite à la ratification de la Déclaration des droits de l'homme et du citoyen en 1789 qui ne s'appliquait qu'aux hommes que les féminins ont commencé à disparaître de l'usage quotidien. (Mathieu - Pierrel 2009 : 111). L'histoire affecte l'emploi des féminins jusqu'à nos jours. Cerquiglini (1999: 10-13) range les substantifs des appellations professionnelles dans deux groupes selon le métier qu'ils désignent - les valorisés et les non valorisés (ou les métiers manuels). Autrefois, nous pouvions nous adresser à une femme exerçant un métier manuel comme cordiere, boutonniere, lavandiere, portière ${ }^{3}$ avec un substantif féminin. Par contre, les féminins des métiers valorisés ne servaient qu'à désigner des épouses.

\section{La France}

La féminisation linguistique en France n'est pas d'ordre systématique. On observe quelques tendances contradictoires. Par exemple, certaines dirigeantEs refusent l'appellation Madame la directrice et préfèrent Madame le directeur. Leurs arguments sont évidemment motivés par l'usage historique, lorsque les féminins des métiers valorisés servaient à nommer des épouses et non les (femmes) titulaires de hauts postes. Les (femmes) directeurs disent que le féminin une directrice n'est acceptable que pour les directrices des écoles. Un autre exemple, plus récent, est tiré de l'article Doit-on appeler bâtonnier ou

\footnotetext{
Les exemples tirés du Livre de la Taille de l'an 1296 et 1297.
} 
bâtonnière une femme à la tête d'un barreau ${ }^{4}$ dans lequel Christiane Féral-Schuhl répond à cette question «Bâtonnier ! [...] La bâtonnière, cela n'a rien à voir, c'est ainsi qu'on dénomme l'épouse du bâtonnier. » C'est un héritage du passé, mais Céline Labrosse met l'accent sur le fait qu'il s'agit en même temps de la réticence des gens.

Le deuxième cas de figure paradoxal sont les dictionnaires. Le Robert, dictionnaire historique de la langue française (2000 : 261) n'introduit pas le féminin du nom auteur affirmant qu'il n'existe pas dans le français utilisé sur le territoire de la France. Par contre, il introduit le féminin auteure dans le français au Québec et les archaïsme français auteuresse, autoresse, authoresse et autrice. Il est illogique de ne reconnaître aucune forme féminine et en présenter cinq en même temps. Même si les féminins archaïques ne sont plus employés, ils prouvent l'existence des formes féminines distinctes.

Suivant les règles et les conseils dans le document Femmes, j'écris ton nom ... Guide d'aide à la féminisation des noms de métiers, titres, grades et fonctions (Cerquiglini 1999), toutes les appellations professionnelles disposent d'un équivalent féminin quelle que soit la façon de sa formation. Ce guide contient la liste détaillée des professions et pour chacune renvoie à une règle concrète pour la mettre au féminin. Les auteurs s'inspiraient, entre autre, du français au Québec, qui est, comme nous le montrerons ci-dessous, beaucoup plus favorable à la féminisation linguistique que le français en Europe. C'est une marque prouvant l'influence entre les différents pays francophones dans le domaine de la féminisation de la langue (Elmiger $2011: 74$ ).

\section{Le Québec}

La féminisation linguistique au Québec n'est pas comparable à celle en France et dans d'autres pays francophones en Europe. La féminisation lexicale a été bien acceptée par le public et les québecois féminisent à chaque fois qu'ils parlent d'une femme et où il est grammaticalement possible d'utiliser le féminin. Aujourd'hui, les linguistes québecois préconisent la féminisation des textes et des discours. Céline Labrosse (2000) met en relief l'importance de la féminisation des textes parce que la langue n'est pas neutre et tout mot est porteur de sens et cela permet d'influencer et de former le comportement social et l'attitude des individus. Les principes de la féminisation des textes consistent à l'application systématique de la féminisation lexicale et aux changements morphosyntaxiques et stylistiques qui en résultent. Il est déconseillé d'employer les masculins génériques. Kristoffer Nyrop (1936 : 71), a écrit par rapport à des masculins génériques : "Après avoir lu que le masculin inclut, englobe et efface le féminin, que le masculin est un neutre, que le nom masculin englobe le féminin, que le féminin est littéralement vu comme dominé par le masculin, que le masculin embrasse le féminin, voilà que Monsieur inclut Madame. »

En 1991, seulement quelques années après que la féminisation (lexicale) ait été introduite en France, l'Office de la langue française au Québec a publié Au féminin : Guide de féminisation des titres de fonction et des textes (Biron et al. 1991). La raison pour laquelle les québecois se sont mis à féminiser des textes reste maintenu - respecter la place des femmes dans la société. Alors qu'au Québec la féminisation avance à grand pas, les tendances dans les pays francophones en Europe sont statiques et ils s'orientent toujours vers la féminisation lexicale.

\footnotetext{
4 La Croix, n. 39165 du 6 janvier 2012.
} 
Il existe plusieurs formes pour féminiser des textes. À l'exception des documents officiels parus au niveau gouvernemental, certains bureaux et entreprises privés inventent leurs propres stratégies de rédaction des textes pour en assurer l'uniformité. Les adversaires de la féminisation des textes argumentent que cela cause une redondance. Les textes deviennent trop longs et moins lisibles.

Voici un exemple des principes de la féminisation des textes utilisés à l'Université de Québec à Montréal :

\begin{tabular}{|l|l|}
\hline Types de texte & Exemple(s) \\
\hline Informatifs & $\begin{array}{l}\text { une étudiante ou un étudiant inscrit } \\
\text { un étudiant ou une étudiante inscrite }\end{array}$ \\
\hline Juridiques & une étudiante, un étudiant inscrit \\
\hline Formulaires & Un-e étudiant-e inscrit-e \\
\hline
\end{tabular}

Tableau 1 : Stratégies de la féminisation des textes à l’Université de Québec à Montréal

\section{La Communauté française de Belgique et la Suisse romande}

Les tendances féministes en linguistique dans ces deux pays francophones européens sont comparables à celles en France. La féminisation lexicale y prévaut aussi.

En Belgique, la féminisation linguistique a été traitée au niveau politique pendant la première moitié des années $90 .^{5}$ Les recommandations publiées dans Mettre au féminin (1994) déconseillent l'usage des masculins génériques, mais les auteurs ne proposent rien pour les remplacer. Marie-Louise Moreau (1999 : 78) juge que la féminisation linguistique ne sera acceptée par le public qu'après une prise de position différente à l'égard de l'égalité des femmes et des hommes dans la société du XXI" siècle.

Vu que la Suisse est une unité fédérative et plurilingue, les approches féministes en linguistique y sont très variées. La féminisation est traitée à trois niveaux politiques fédéral, cantonal et communal. Les essais les plus efficaces ont été réalisés par la majorité des cantons qui insistent sur la féminisation systématique. Cependant, il en reste quelques-uns qui ne s'y intéressent pas du tout. La Délégation à la langue française de Suisse romande représentée par Jean-François De Pietro et Marie-José Béguelin (1999: 43) soutient le fait que les mesures linguistiques sont inutiles et qu'il faut attendre que les femmes et les hommes soient reconnus égaux pour pouvoir commencer à résoudre la féminisation de la langue.

Ce qui est sûr, c'est qu'il reste encore beaucoup de travail à accomplir, pas seulement en Suisse romande et en Communauté française de Belgique, pour que les femmes et les hommes soient égaux dans la société et dans la langue. La langue fait partie intégrante de la société et elle ne devrait pas ignorer le développement social.

Depuis l'accès des femmes à de nouvelles fonctions et devant le désir de celles-ci de voir leurs désignations refléter cette nouvelle réalité, il est recommandé d'utiliser les formes féminines des titres de fonction. ${ }^{6}$

\section{Les règles de la féminisation linguistique}

La formation des féminins est étudiée en détails dans les quatre pays. La majorité des règles coïncident ou les pays s'inspirent l'un de l'autre. Cependant, il y a des règles qui

5 Décret du 21 juin 1993 relatif à la féminisation des noms de métier, fonction, grade ou titre.

6 Gazette officielle du Québec, le 28 juillet 1979. 
ne correspondent pas dans les pays francophones où il existe plusieurs formes féminines d'une seule appellation professionnelle. Ces règles sont publiées sous des formes variées, elles sont plus ou moins complexes et servent à simplifier et intensifier la formation et l'usage des noms de métier féminins. Les documents écrits traitant de féminisation linguistique peuvent être classés en deux groupes :

\begin{tabular}{|l|l|}
\hline Types de document & Exemples \\
\hline $\begin{array}{l}\text { Documents spécialisés (savants et } \\
\text { pragmatiques) }\end{array}$ & grammaires, guides - manuels, dictionnaires, etc. \\
\hline Documents politiques & circulaires, lois, décrets, directives, etc. \\
\hline
\end{tabular}

Tableau 2: Classement des documents traitant de féminisation linguistique

Daniel Elmiger (2008 : 206) caractérise ces documents comme « les texte[s] à visée évaluative et normative ayant pour l'objet l'emploi public de la langue, et plus précisément la manière dont on fait référence à des femmes et à des hommes. " Sa définition couvre les deux groupes. Pourtant, la différence entre eux est notable même si l'objet est semblable. Alors que les documents appartenant au premier groupe proposent des choses sans rien imposer, les documents du deuxième groupe insistent sur l'emploi des féminins dans des situations de communication concrètes, par exemple l'administration et la législation.

Actuellement, il n'existe pas encore un document universel sur la féminisation de la langue française. Les linguistes ne dépassent pas les limites de leur propre pays dans les recherches d'où de nombreuses différences des règles et des approches de la féminisation. Dans le tableau suivant, nous démontrons sur quelques exemples tirés des documents de la féminisation linguistique que les approches linguistiques et sociolinguistiques ne sont pas unanimes en milieu francophone. Si l'on regarde l'année de parution de chaque publication et le sujet traité, on s'aperçoit que c'est le Québec qui est allé le plus loin et que la féminisation des textes ne trouve pas encore dans la langue française en Europe.

\begin{tabular}{|l|c|c|c|}
\hline \multicolumn{1}{|c|}{ (Provenance) Titre } & \multirow{2}{*}{$\begin{array}{c}\text { Année } \\
\text { de parution }\end{array}$} & f. lexicale & f. des textes \\
\cline { 3 - 4 } & 1979 & + & \\
\hline $\begin{array}{l}\text { (Q) Féminisation des titres (les } \\
\text { recommandations publiées dans la Gazette } \\
\text { officielle du Québec, le 28 juillet 1979) }\end{array}$ & 1988 & & + \\
\hline $\begin{array}{l}\text { (Q) Guide pour la rédaction de textes non } \\
\text { sexistes }\end{array}$ & 1991 & + & + \\
\hline $\begin{array}{l}\text { (Q) Au féminin : guide de féminisation des } \\
\text { titres de fonctions et des textes }\end{array}$ & 1997 & + & + \\
\hline $\begin{array}{l}\text { (Q) Guide de féminisation ou la } \\
\text { représentation des femmes dans les textes }\end{array}$ & 1991 & + & + \\
\hline $\begin{array}{l}\text { (S) La formulation non sexiste des textes } \\
\text { législatifs et administratifs }\end{array}$ & + & + \\
\hline
\end{tabular}


La féminisation linguistique en milieu francophone

\begin{tabular}{|l|c|c|c|}
\hline $\begin{array}{l}\text { (S) Le nouveau dictionnaire féminin- } \\
\text { masculin des professions, des titres et des } \\
\text { fonctions }\end{array}$ & 1999 & + & + \\
\hline $\begin{array}{l}\text { (F) Rapport sur la féminisation des noms de } \\
\text { métier, fonction, grade ou titre }\end{array}$ & 1998 & + & \\
\hline $\begin{array}{l}\text { (F) Femme, j' écris ton nom ... Guide d'aide } \\
\text { à la féminisation des noms de métiers, titres, } \\
\text { grades et fonctions }\end{array}$ & 1999 & + & partiellement \\
\hline $\begin{array}{l}\text { (B) Mettre au féminin. Guide de fémnisation } \\
\text { des noms de métier, fonction, grade ou titre }\end{array}$ & 1994 & + \\
\hline
\end{tabular}

Tableau 3 : Documents traitant de féminisation linguistique dans les pays francophones

Au Québec, le premier ouvrage spécialisé a été publié en 1979 tandis qu'en Europe ce n'était qu'au cours des années 80 et 90 . Le Québec est aussi le premier pays francophone à s'intéresser au langage sexiste et à la féminisation des textes (1988). C'est aussi à cause de ce décalage temporel qu'il y a des différences. Pour les illustrer, nous introduisons deux cas de figures :

\begin{tabular}{|l|l|}
\hline un professeur / une professeure & Q, S \\
\hline un professeur / une professeur $\varnothing$ & B, F \\
\hline
\end{tabular}

Tableau 4 : Différences (exemple 1 )

En France et en Belgique, la formation du féminin ne se fait qu'avec le déterminant. Les québecois et les suisses ajoutent un - $e$ au masculin pour spécifier le genre aussi à l'écrit. Le français dispose aujourd'hui d'un troisième féminin, une professeuse. Même si le verbe professer (enseigner en qualité de professeur) n'a pas un - $t$ dans sa terminaison, la forme une professeuse a été dérivée selon la règle comme l'explique Cerquiglini (1999 : 24) : s'il existe un rapport sémantique direct entre le verbe comportant un - $t$ dans sa terminaison et le nom masculin se terminant en -eur, on peut former le féminin à l'aide du suffixe -euse ; acheter - un acheteur $\rightarrow$ une acheteuse.

\begin{tabular}{|l|c|}
\hline un directeur / une directrice & $\mathrm{Q}$ \\
\hline un directeur / une directrice* & $\mathrm{F}$ \\
\hline
\end{tabular}

Tableau 5 : Différences (exemple 2)

La forme une directrice se trouve dans les dictionnaires français, québécois, suisses et belges mais elle n'est systématiquement employée que par les québécois. En France, les femmes préfèrent l'appellation un directeur affirmant que celle-ci est plus prestigieuse.

\section{Conclusion}

La féminisation linguistique est un sujet d'actualité depuis des années. Son objectif principal est de refléter la réalité sociale dans la langue, à savoir l'accès des femmes à toutes les professions. La société et ses membres changent tous les jours ce qui exige aussi une flexibilité au niveau de la langue. L'application des changements sociaux à la langue est un long processus qui n'est pas toujours mené à bien. En effet, une forme féminine codifiée par les grammairiens et mentionnée dans les dictionnaires n'est pas toujours accep- 
tée par les sujets parlants (un directeur $\mathrm{x}$ une directrice). Le développement de l'usage des féminins n'est pas unanime en milieu francophone. Il nous paraît indispensable de créer une analyse comparative que tout le monde francophone pourra utiliser.

\title{
Bibliographie
}

Biron, Monique (1991), Au féminin. Guide de féminisation des titres de fonction et des textes, Québec : Office de la langue française.

Bouchard, Pierre - Guilloton, Noëlle - Vachon L'Heureux, Pierrette - De Pietro, Jean-François - Béguelin, Marie-José - Mathieu, Marie-Josèphe - Moreau, Marie-Louise (1999), La féminisation des noms de métiers, fonctions, grades ou titres au Québec, en Suisse romande, en France et en Communauté française de Belgique, Louvain-la-Neuve : Duculot.

Cerquiglini, Bernard - Becquer, Anne-Marie et al. (1999), Femme, j'écris ton nom ... Guide d'aide à la féminisation des noms de métiers, titres, grades et fonctions, Paris : Institut national de la langue française.

ElMiger, Daniel (2008), La féminisation de la langue en français et an allemand. Querelle entre spécialistes et réception par le grand public, Paris: Honoré Champion.

Elmiger, Daniel (2011), «Féminisation de la langue française : une brève histoire des positions politiques et du positionnement linguistique », in : DuCHÊNE, Alexandre - MoÏse, Claudine (éds.), Langage, genre et sexualité, Québec : Les éditions Nota Bene, 71-89.

Labrosse, Céline (1996), Pour une grammaire non sexiste, Montréal : Les éditions du remue ménage.

Labrosse, Céline (2002), Pour une langue française non sexiste, Montréal : Les éditions des Intouchables.

LARIVIÈRE, Louise-Laurence (2000), Pourquoi en finir avec la féminisation linguistique ou à la recherche des mots perdus, Montréal : Éditions du Boréal.

Mathieu, Marie-Josèphe - Pierrel, Jean Marie (2009), " La féminisation dans la langue », in : Guittienne, Lucile - Prost, Marlène (éds.), Homme-femme, de quel sexe êtes-vous ?, Nancy : Presses universitaires de Nancy, 111-122.

NYrop, Kristoffer (1936), Grammaire historique de la langue française, Copenhague: Gyldendal.

\author{
Martin Pleško \\ Katedra romanistiky \\ Filozofická fakulta \\ Univerzita Palackého v Olomouci \\ Křížkovského 8 \\ 77180 Olomouc \\ République tchèque \\ martin.plesko@gmail.com
}

